Published in final edited form as:

Clin Cancer Res. 2018 October 15; 24(20): 5165-5177. doi:10.1158/1078-0432.CCR-18-0279.

\title{
Loss of E-cadherin enhances IGF1-IGF1R pathway activation and sensitizes breast cancers to anti-IGF1R/InsR inhibitors
}

\author{
Alison M. Nagle ${ }^{1,2}$, Kevin M. Levine ${ }^{1,3}$, Nilgun Tasdemir ${ }^{1,2}$, Julie A. Scott ${ }^{1,2}$, Kara \\ Burlbaugh $^{2}$, Justin Kehm ${ }^{2}$, Tiffany A. Katz ${ }^{1,2,4}$, David N. Boone ${ }^{2,5}$, Britta M. Jacobsen ${ }^{6}$, \\ Jennifer M. Atkinson ${ }^{1,2}$, Steffi Oesterreich ${ }^{1,2,3}$, Adrian V. Lee $^{1,2,7}$ \\ 1.Dept. of Pharmacology and Chemical Biology, University of Pittsburgh, Pittsburgh, PA \\ 2.Women's Cancer Research Center, UPMC Hillman Cancer Center, Magee Women's Research \\ Institute, Pittsburgh, PA \\ 3.Dept. of Pathology, University of Pittsburgh, Pittsburgh, PA \\ 4. The Center for Precision Environmental Health, Dept. of Molecular and Cellular Biology, Baylor \\ College of Medicine, Houston, TX (current) \\ 5.Dept. of Biomedical Informatics, University of Pittsburgh, Pittsburgh, PA \\ 6.Dept. of Pathology, University of Colorado Anschutz Medical Campus, Aurora, CO \\ 7.Dept. of Human Genetics, University of Pittsburgh, Pittsburgh, PA
}

\begin{abstract}
Purpose: Insulin-like growth factor 1 (IGF1) signaling regulates breast cancer initiation and progression and associated cancer phenotypes. We previously identified E-cadherin $(\mathrm{CDH} 1)$ as a repressor of IGF1 signaling and in this study examined how loss of E-cadherin affects IGF1R signaling and response to anti-IGF1R/insulin receptor (InsR) therapies in breast cancer.
\end{abstract}

Experimental Design: Breast cancer cell lines were used to assess how altered E-cadherin levels regulate IGF1R signaling and response to two anti-IGF1R/InsR therapies. In situ proximity ligation assay (PLA) was used to define interaction between IGF1R and E-cadherin. TCGA RNAseq and RPPA data was used to compare IGF1R/InsR activation in estrogen receptor positive (ER +) invasive lobular carcinoma (ILC) and invasive ductal carcinoma (IDC) tumors. ER+ ILC cell lines and xenograft tumor explant cultures were used to evaluate efficacy to IGF1R pathway inhibition in combination with endocrine therapy.

Results: Diminished functional E-cadherin increased both activation of IGF1R signaling and efficacy to anti-IGF1R/InsR therapies. PLA demonstrated a direct endogenous interaction between IGF1R and E-cadherin at points of cell-cell contact. Increased expression of IGF1 ligand and levels of IGF1R/InsR phosphorylation were observed in E-cadherin deficient ER+ILC compared to IDC tumors. IGF1R pathway inhibitors were effective in inhibiting growth in ER+ ILC cell

Corresponding author: Adrian V. Lee (leeav@upmc.edu), Magee Women’s Research Institute, A412, 204 Craft Ave Pittsburgh, PA 15213, Phone: 412-641-7557; Fax: 412-641-2458.

The authors declare no potential conflicts of interest. 
lines and synergized with endocrine therapy and similarly IGF1R/InsR inhibition reduced proliferation in ILC tumor explant culture.

Conclusions: We provide evidence that loss of E-cadherin hyperactivates the IGF1R pathway and increases sensitivity to IGF1R/InsR targeted therapy, thus identifying the IGF1R pathway as a potential novel target in E-cadherin deficient breast cancers.

\section{Keywords}

breast cancer; invasive lobular carcinoma; insulin-like growth factor 1 receptor (IGF1R); Ecadherin; growth factor signaling

\section{INTRODUCTION}

IGF1 is a circulating endocrine hormone that is a major regulator of organismal growth and development ${ }^{1}$. IGF1, in combination with estrogen, is essential for normal mammary gland development, and this pathway is deregulated in the initiation and progression of breast cancer $^{2-5}$. Many studies, including from our laboratory, have shown the ability of the IGF1 receptor (IGF1R) to promote mammary tumorigenesis and metastasis ${ }^{6-9}$. Additionally, we showed that when constitutively activated, IGF1R transformed mammary epithelial cells, increased migration and invasion, and induced epithelial to mesenchymal transition (EMT) via the NFkB pathway and upregulation of Snail ${ }^{6,10}$.

Based on these observations, both small molecule tyrosine kinase inhibitors and monoclonal antibodies against IGF1R were tested in clinical trials in breast cancer. Unfortunately, although as many as $50 \%$ of breast tumors express IGF1R ${ }^{11}$, these trials only identified a small subset of patients showing a therapeutic response to IGF1R targeted therapy, suggesting that predictive biomarkers are required to identify which patients' tumors will be responsive ${ }^{12-15}$.

We previously developed an IGF1-signature (IGF-sig) based on microarray analyses, and more recently reported a novel computational method to identify putative biomarkers of IGF1 signaling using a systems biology approach ${ }^{16}$. The latter was based on a proteomic screen using reverse phase protein array (RPPA) on 21 breast cancer cell lines stimulated with IGF1 over a time course ${ }^{17}$. This computational model identified E-cadherin as a putative regulator of IGF1 signaling, and data in the present study indicate that loss of Ecadherin expression can directly increase IGF1R pathway activation and associated phenotypes in breast cancer. Insight into how E-cadherin regulates IGF1R is necessary to aid in our understanding of the oncogenic signaling network, specifically because the loss of Ecadherin i) is implicated in the ability of tumor cells to escape the primary tumor to potentially seed metastatic lesions and ii) is transcriptionally repressed and/or genetically lost in subsets of breast tumors ${ }^{18-22}$.

One such subtype of breast cancer with diminished E-cadherin expression is invasive lobular breast carcinoma (ILC), accounting for 10-15\% ( 30,000 cases/year in the US) of total breast cancer cases. ILC is defined by the loss of functional E-cadherin ( $C D H 1)$, which occurs in $95 \%$ of ILC due to truncating mutations, loss of heterozygosity, and transcriptional 
repression ${ }^{23,24}$. Due to the loss of E-cadherin protein, ILC cells grow in linear patterns throughout the breast tissue, lacking the ability to form adherens junctions, in contrast to the solid mass growth of the most frequent subtype of breast cancer, invasive ductal breast carcinoma (IDC) $)^{25}$. Interestingly, one of the most IGF1 responsive cell lines in our abovereferenced proteomic data set was a human ILC cell line, MDA-MB-134-IV, that lacks Ecadherin protein expression and cell-cell junctions ${ }^{17}$. In this study, we characterize the regulation of IGF1R by E-cadherin and provide evidence that inhibition of IGF1R/insulin receptor (InsR) in E-cadherin deficient breast cancers could potentially serve as an effective therapeutic strategy.

\section{MATERIALS AND METHODS}

\section{Cell culture}

Cell lines were authenticated (most recent date listed [ ]) by the University of Arizona Genetics Core and mycoplasma tested (Lonza \#LT07-418). Lab stocks were made following authentication and used for this study. MCF-7 (ATCC; DMEM+10\% FBS [06/29/16]), T47D (ATCC; RPMI+10\% FBS [02/08/17]), ZR75.1 (ATCC; RPMI+10\% FBS [10/13/16]), MDAMB-231 (ATCC; DMEM+10\% FBS [10/13/16]), MDA-MB-134-VI (ATCC; 50/50 DMEM/ L15+10\% FBS [02/08/17]), SUM44PE (Asterand; DMEM/F12+2\% CSS with 5ug/ml insulin, $1 \mathrm{ug} / \mathrm{ml}$ hydrocortisone, $5 \mathrm{mM}$ ethanolamine, $5 \mathrm{ug} / \mathrm{ml}$ transferrin, $10 \mathrm{nM}$ triodothyronime, and 50nM sodium selenite [02/08/17 - no reference profile exists in database]), and BCK4 ${ }^{26}$ (MEM+5\% FBS with $1 \mathrm{nM}$ insulin and 1× NEAA [10/13/16 - no reference profile exists in database) cells were cultured with indicated media conditions.

\section{Transient siRNA transfection}

Cells were reverse transfected with $25 \mathrm{nM}$ final concentration of siGENOME human SMARTpool control siRNA (Dharmacon \#D-001206) or siGENOME human SMARTpool CDH1 siRNA (Dharmacon \#M-003877-02) using Lipofectamine RNAiMAX (Invitrogen \#13778) protocol for 48 hours. For IGF1 (GroPep BioReagents \#CU100) stimulation, cells were serum starved overnight and pulsed with IGF1 (1nM, 10nM, or 100nM) for 10 minutes.

\section{Stable shRNA infection}

Stable CDH1 knockdown T47D cells were generated using a retro-viral infection of Renilla control (shSCR [5' TGCTGTTGACAGTGAGCGCAGGAATTATAATGCTTATCTATA GTGAAGCCACAGATGTATAGATAAGCATtATAATTCCTATGCCTACTGCCTCGGA]) and two CDH1 (sh-1 [5' TGCTGTTGACAGTGAGCGCAAGTGTGTtCATTAATGTTTATAGTGAAGCC ACAGATGTATAAACATtAATGAACACACTTATGCCTACTGCCTCGGA] and sh-2 [5' tgCtgt TGACAGTGAGCGACCGGGACAACGTTTATTACTATAGTGAAGCCACAGATGTATAGTAATAAACGTTG TCCCGGGTGCCTACTGCCTCGGA]) short-hairpin RNAs (shRNA). Cells were selected with growth media supplemented with 1ug/ml Puromycin (Life \#A11138-03). 


\section{Plasmid DNA overexpression}

MDA-MB-231 cells were stably transfected using FUGENE6 with empty or hE-cadherinpcDNA3 vector (Addgene \#45769) using 15ug DNA per $10 \mathrm{~cm}$ plate of cells. Cells were selected in growth media supplemented with 800ug/ml G418 (Invitrogen \#10131-035).

\section{Immunoblotting}

Samples for immunoblot analysis were collected using RIPA buffer (50mM Tris pH 7.4, $150 \mathrm{mM} \mathrm{NaCl}, 1 \mathrm{mM}$ EDTA, $0.5 \%$ Nonidet P-40, 0.5\% NaDeoxycholate, $0.1 \%$ SDS, $1 \times$ HALT cocktail [Thermo Fisher \#78442]) and standard immunoblot technique was followed. Membranes were blocked in Odyssey PBS Blocking Buffer (LiCor \#927-40000), and incubated in primary antibodies overnight: pIGF1R/InsR (Cell Signaling \#3918; 1:500), IGF1R $\beta$-subunit (Cell Signaling \#3027; 1:1000), pAkt S473 (Cell Signaling \#4060; 1:1000), total Akt (Cell Signaling \#9272; 1:1000), E-cadherin (BD Biosciences \#610182; 1:1000), and $\beta$-actin (Sigma \#A5441; 1:5000). Membranes were incubated in LiCor secondary antibodies for 1 hour (anti-rabbit 800CW [LiCor \#926-32211]; anti-mouse 680LT [LiCor \#925-68020]; 1:10,000), and imaged with Odyssey Infrared Imager. Super signal femto detection chemiluminescence (Thermo \#34094) was used to detect total IGF1R levels in ZR75.1 cells due to low expression level.

\section{IGF1-induced cell cycle and viability analysis}

For cell cycle: MCF-7 and ZR75.1 cells were reverse transfected as described above, serum starved for approx. 30 hours, and pulsed with 10nM IGF1 for 17 hours. Cells were fixed in $70 \% \mathrm{EtOH}$ for 30 minutes at $4^{\circ} \mathrm{C}$ and RNA digested using 50ng/ul RNase A (Qiagen \#1007885) for 15 minutes at $37^{\circ} \mathrm{C}$. DNA content was then stained using 50ng/ul propidium iodide (Sigma \#P4170) for 30 minutes at $4^{\circ} \mathrm{C}$. Cell cycle profiles were analyzed using the BD LSRII flow cytometer and analyzed using the FACS DIVA software. The statistical difference in percent of cells in S- or G2/M phase in IGF1 treated cells over vehicle control in experimental groups was evaluated using a two-tailed student's t-test $(\mathrm{p}<0.05)$.

For viability: T47D shSCR and shCDH1 \#1 and \#2 cells were plated in serum-free media in 96 well plates $(9,000$ cells/well) and then stimulated with IGF1 (10nM) for 6 days. The FluoReporter Blue Fluorometric dsDNA Quantitation Kit was used to measure DNA content. Statistical difference in Hoechst fluorescence in IGF1 treated cells over vehicle control in each cell line was evaluated using a two-tailed student's t-test $(\mathrm{p}<0.05)$.

\section{Immunofluorescence and Proximity ligation assay (PLA)}

Cells were plated on coverslips and fixed in $4 \%$ paraformaldehyde for 30 minutes at $37^{\circ} \mathrm{C}$. Coverslips were permeabilized for 1 hour using PBS+0.3\% Triton X-100. For immunofluorescence, coverslips were blocked in $\mathrm{PBS}+5 \%$ goat serum, incubated in primary antibody overnight (total IGF1R $\beta$-subunit [Cell Signaling \#3027; 1:300] and E-cadherin [BD Biosciences \#610182; 1:100]), followed by Alexa Fluor secondary antibody incubation for 1 hour (anti-rabbit Alexa Fluor 488 [Life Technologies \#A11070] and anti-mouse Alexa Fluor 546 [Life Technologies \#A11018]; 1:200). For in situ proximity ligation assay, 
coverslips were processed using the Duolink Red mouse/rabbit kit using the protocol provided (Sigma \#DUO92101) with the antibody dilutions above. The ratio of puncta/nuclei for each experimental condition was calculated by counting all puncta and nuclei in five 60x images. One-way ANOVA was used to compare the ratios between the experimental conditions (VHC, 30m, 6hr, 24hr). Confocal microscopy was used for imaging.

\section{Dose response growth assays and synergy measurements}

MCF-7 and ZR75.1 cells were reverse transfected with control or CDH1 siRNA as described above into 96-well plates $(9,000$ cells/well) in 100ul of media/well. Cells were treated with $3 \times$ vehicle (DMSO), OSI-906 (Selleckchem \#S1091) or BMS-754807 diluted in 50ul of media for a final volume in each well of $150 \mathrm{ul}$ ( $\mathrm{n}=6$ per concentration). Plates ( $2 \mathrm{D}$ and ultralow attachment [ULA; Corning \#3474]) were collected on day 6 and viability was measured using CellTiter Glo Viability assay (Promega \#G7572). EC 50 values for viability were calculated by non-linear regression and statistical differences evaluated using sum-ofsquares Global $\mathrm{f}$-test $(\mathrm{p}<0.05)$. For synergy experiments, SUM44PE and MDA-MB-134 cells were plated in 96-well ULA plates (18,000 cells/well) in 100ul of media/well. Cells were treated with 6x vehicle (DMSO), OSI-906, BMS-754807, or BEZ235 (Selleckchem \#S1009) diluted in 25ul of media such that the combination of two drugs resulted in 150ul of total volume in each well ( $\mathrm{n}=2$ per experiment). Synergy was calculated using the MedianEffect Principle and Combination Index-Isobologram Theorem (Chou-Talalay) ${ }^{27}$. Combination index values for ED50, ED75, ED90 are shown as a mean \pm SEM from $n=3$ independent experiments.

\section{In vivo ILC xenograft growth and explant culturing}

MDA-MB-134 cells $\left(5 \times 10^{6}\right.$ cells $)$ and BCK4 cells $\left(5 \times 10^{6}\right.$ cells $)$ were injected into the right inguinal mammary fat pads of 7-8 week old NOD.Cg-Prkdcscid Il2rgtm1Wjl/SzJ (NSG; The Jackson Laboratory [MM134]) and CB17.Cg-PrkdcsidLystbg-J/Crl (SCID-beige; Charles River [BCK4]) respectively (implanted with $0.36 \mathrm{mg}$ 90-day slow release estradiol pellets [Innovative Research of America \#SE-121]) and grown to a tumor volume of $350 \mathrm{~mm}^{3}$. Tumors were collected, minced into $1-2 \mathrm{~mm}^{3}$ chunks of tumor tissue, and plated onto Vetspon Absorbable Hemostatic Gelatin sponges (Patterson Veterinary \#07-849-4032) in 12-well tissue culture plates containing $1.5 \mathrm{mls}$ of explant media (DMEM/F12+10\% FBS with $10 \mathrm{mM}$ HEPES, $1 \mathrm{mg} / \mathrm{ml}$ BSA, $10 \mathrm{ug} / \mathrm{ml}$ insulin, $10 \mathrm{ug} / \mathrm{ml}$ hydrocortisone, $1 \times$ antibioticantimycotic solution [Thermo Fisher \#15240-062]). Media was treated with vehicle or 1uM BMS-754807 for 72 hours. Tissue was collected by formalin fixation followed by paraffin embedding. Sections were stained for Ki67 (Dako \#M7240; 1:100) using standard immunohistochemistry technique. Nuclei were quantified by counting all clearly defined nuclei within each tissue section $(n=3-6)$. Two-tailed student's t-test was used to determine statistical difference between vehicle and BMS-754807 treatment $(\mathrm{p}<0.05)$.

\section{In silico analysis}

TCGA RNA-seq expression data were downloaded as transcripts per million (TPM) from the Gene Expression Omnibus database (GEO: GSE62944) and $\log 2(\mathrm{TPM}+1)$ for gene-level results were used. TCGA Reverse Phase Protein Array (RPPA) data were downloaded as median-normalized, batch-corrected expression values from TCPA (Level 4, version 4.0). 
ER+ IDC ( $\mathrm{n}=417)$ and ILC $(\mathrm{n}=137)$ samples with both RNA-Seq and RPPA data were used for all analyses. Mann-Whitney U tests were used to compare expression, Spearman's rho to compare correlations, and a chi-square test to compare proportions between ILC and IDC tumors. All were calculated using R (version 3.4.1). The median expression values for IGF1 and pIGF1R/InsR (Y1135/1136 [Cell Signaling \#3024]) across ER+ IDC and ILC tumors $(\mathrm{n}=554)$ were used as cutoffs for Figure 4H-I. IGF1R and InsR mRNA levels in MCF-7, T47D, ZR75.1, SUM44PE, MM134, and MM330 cells were plotted using publicly available RNAseq (log2 FKPM) data from Marcotte et al. 2006. Cell (PMID:26771497; accession numbers: GSE73526, GSE74702).

\section{RESULTS}

\section{Loss or inhibition of E-cadherin results in enhanced IGF1R activity}

To validate our previously published data ${ }^{17}$ and to further understand the regulation of the IGF1 signaling pathway by E-cadherin, we silenced E-cadherin $(C D H I)$ by siRNA knockdown in a panel of three estrogen receptor (ER)-positive IDC cell lines and then stimulated with a dose series of IGF1 $(0,1,10,100 \mathrm{nM})$. MCF-7, ZR75.1, and T47D Ecadherin knockdown (siCDH1) cells showed enhanced sensitivity to IGF1 compared to the scramble control (siSCR) cells, most notable at the 1nM dose of IGF1, resulting in increased phosphorylation of IGF1R/InsR (phosphorylation of both receptors detected by antibody due to high sequence homology in kinase domains) and Akt (Fig 1A-C). Interestingly, ZR75.1 cells responded with enhanced pIGF1R/InsR in siCDH1 cells, however the increase in pAkt appears unchanged following IGF1 stimulation, suggesting an independent effect of Ecadherin loss on Akt signaling (Fig 1B). We also compared gene expression of IGF1R and InsR in our panel of cell lines using publicly available RNAseq data due to the abundant crosstalk between the IGF and insulin signaling pathways ${ }^{28}$. Expression of IGF1R was higher than InsR in all cell lines, except for ZR75.1, suggesting that a larger proportion of IGF1-induced signaling may be driven through IGF1R rather than InsR (Fig S1). ZR75.1 cells may rely more heavily on InsR to initiate IGF1 signaling.

As a complementary approach to siRNA knockdown, we inhibited E-cadherin function in MCF-7 cells using the HECD-1 monoclonal antibody that binds the extracellular domain of E-cadherin and prevents adherens junction formation. Similar to the knockdown of Ecadherin, HECD-1 treated cells showed increased IGF1R/InsR and Akt phosphorylation compared to control (Fig 1D). Additionally, we evaluated confluency-dependent IGF1R signaling to understand the effect of increased cell-cell contacts. A confluent monolayer of MCF-7 cells lost the ability to initiate phosphorylation of IGF1R/InsR upon ligand stimulation compared to a sub-confluent monolayer (approx. 40-50\%), however, the knockdown of E-cadherin rescued signaling in both confluency conditions (Fig 1E).

We evaluated the functional effect of enhanced IGF1 signaling on the cell cycle profile in MCF-7 and ZR75.1 cells with reduced E-cadherin. CDH1 knockdown cells showed a significant increase ( $\mathrm{p}=0.03$ and $\mathrm{p}=0.0005$, respectively) in the percentage of cells progressing into the $\mathrm{S}$ - and G2/M-phases of the cell cycle following IGF1 treatment compared to siSCR cells (Fig 1F). Similarly, slight increases in IGF1-induced cell viability in siCDH1 compared to siSCR in T47D cells were observed (Fig S2). 
We overexpressed E-cadherin in MDA-MB-231 cells, an ER-negative IDC cell line with undetectable E-cadherin protein by immunoblot to determine if overexpression represses signaling. E-cadherin overexpressing cells demonstrated decreased phosphorylation of IGF1R/InsR and Akt compared to empty vector control cells, and significantly less cell cycle progression in response to IGF1 stimulation ( $\mathrm{p}=0.011$; Fig S3).

\section{Loss of E-cadherin enhances sensitivity to IGF1R/InsR inhibition}

Due to the enhanced sensitivity of E-cadherin knockdown cells to IGF1 stimulation, we determined if loss of E-cadherin in MCF-7 and ZR75.1 cells also increased sensitivity to the IGF1R/InsR ATP-competitive small molecule inhibitors, OSI-906 (OSI) and BMS-754807 (BMS). Due to the highly homologous kinase domains of IGF1R and InsR, small molecule inhibitors target both receptors with equal potency. In addition to 2D adherent culture, ultralow attachment suspension growth (ULA) was examined, since we observed increased cell viability in E-cadherin knockdown cells under these conditions (Tasdemir et al, manuscript in preparation), possibly due to the reported annoikis resistance of cells lacking E-cadherin expression ${ }^{29}$. MCF-7 siCDH1 cells displayed significantly decreased viability in response to OSI treatment, compared to siSCR cells in both $2 \mathrm{D}$ ( $\mathrm{p}<0.0001$; Fig $2 \mathrm{~A})$ and ULA $\left(\mathrm{p}=0.0003\right.$; Fig 2B) growth conditions resulting in a shift in the $\mathrm{EC}_{50}$. Additionally, ZR75.1 siCDH1 cells showed significantly decreased viability and a shift in the $\mathrm{EC}_{50}$ when grown in ULA ( $p<0.0001$; Fig S4) in response to OSI treatment, but not in the 2D growth condition. Similarly, MCF-7 siCDH1 cells showed decreased viability in response to BMS compared to siSCR cells the ULA growth condition ( $\mathrm{p}<0.0001$ ), but no significant difference in 2D (Fig 2C-D). We do not yet understand the differential response of MCF-7 siSCR and siCDH1 cells to OSI-906 and BMS-754807 grown in 2D, but think this may be a result of differing potencies or potential off-target effects of the inhibitors. Overall, these data suggest that the loss of E-cadherin enhances breast cancer cell sensitivity to IGF1R/InsR inhibition. We also tested the growth response of MCF-7 siSCR and siCDH1 cells treated with ICI 182,780 (ICI), a selective estrogen receptor downregulator (SERD), and observed no statistical difference in $\mathrm{EC}_{50}$ suggesting that the loss of E-cadherin does not generally sensitize cells to all small molecule drug treatments (Fig S5).

\section{IGF1R and E-cadherin directly interact in ER+ breast cancer cells resulting in recruitment of IGF1R to adherens junctions}

To understand how E-cadherin regulates IGF1R, we assessed whether IGF1R and Ecadherin directly interact in breast cancer cells using in situ proximity ligation assay (PLA). The sensitivity and specificity of PLA allows for detection of endogenous interacting proteins within proximity of no further than 40nm. PLA showed that IGF1R and E-cadherin directly interact in both MCF-7 and T47D cells, as shown by the red fluorescent puncta (Fig 3A-B [high magnification], Fig S6A-B [low magnification]). To demonstrate the specificity of the detection, we used MCF-7 knockdown cells lacking E-cadherin (siCDH1) or IGF1R (siIGFR) as negative controls and observed the red fluorescent puncta signal greatly diminished (Fig 3C-D, Fig S6C-D, Fig S7A-C). Additionally, secondary antibody specificity was confirmed by using each primary antibody alone and a no primary antibody control and did not detect significant levels of PLA puncta over background (Fig S7D-F). The interaction between IGF1R and E-cadherin following IGF1 stimulation was examined using 
PLA. In MCF-7 cells, IGF1 treatment caused a significant decrease in number of fluorescent puncta ( $\mathrm{p}=0.003$ ), suggesting that the interaction between the two proteins needs to be disrupted for proper IGF1R function (Fig 3E-I [high magnification], Fig S6E-H [low magnification]), possibly explaining why siCDH1 cells have an increased IGF1R signaling capacity compared to control cells. Of note, the serum-starvation required for assessing the effect of IGF1 stimulation on the interaction between IGF1R and E-cadherin (Fig 3E) reduced the number of puncta compared to full-serum conditions (Fig 3A).

We stained MCF-7 cells for endogenous IGF1R and E-cadherin and determined that IGF1R and E-cadherin co-localize to adherens junctions. Interestingly, co-localization was prominent at the points of cell-cell contact, and noticeably absent or reduced on portions of the membrane where there was no cell-cell contact (Fig 3J). This suggests that although both exhibit membranous staining, E-cadherin recruits IGF1R to adherens junctions where expression is increased, perhaps to sequester the receptor as a mechanism of signaling repression. Supporting this, the number of PLA puncta indicative of IGF1R/E-cadherin complex formation increased in MCF-7 cells within a colony, compared to a single cell, localized to both the cell membrane and intracellular compartments (Fig S8). Upon knockdown of E-cadherin the expression pattern of IGF1R appears to redistribute equally to the entire cell membrane (Fig 3K) supporting the idea that E-cadherin influences and regulates IGF1R localization.

\section{Invasive lobular breast cancers (ILC) display enhanced IGF1-IGF1R pathway activation}

Because knockdown or inhibition of E-cadherin induces hyperactivity of the IGF1R pathway in cell line models, we investigated whether IGF1R pathway activity is also hyperactivated in ILC, a subtype of breast cancer that accounts for 10-15\% of all breast cancer cases and is molecularly classified by its genetic loss of E-cadherin ${ }^{23}$. Because $90-95 \%$ of ILC tumors are ER+, we focused on this cohort ${ }^{23}$. IGF1R expression and localization was examined in the ER+ ILC cell lines: SUM44PE (Fig4A), MDA-MB-134 (MM134; Fig 4B) and BCK4 (Fig 4C). IGF1R staining was membranous similar to that observed in MCF-7 siCDH1 cells (Fig 3F). As expected, ILC cells showed a lack of membranous E-cadherin staining (Fig 4AC).

To compare IGF1R activity in ER+ ILC and IDC tumors, CDH1 and IGF1/2 ligand mRNA expression, and IGF1R/InsR phosphorylation were examined using RNA-sequencing and Reverse Phase Protein Array data from The Cancer Genome Atlas (TCGA). Concurrent with a decrease in CDH1 mRNA expression ( $\mathrm{p}=9.06 \mathrm{e}-52$; Fig 4D), IGF1 ligand ( $\mathrm{p}=1.3 \mathrm{e}-15$; Fig 4E), and IGF2 ligand ( $\mathrm{p}=5.36 \mathrm{e}-06$; Fig4F) mRNA expression, and pIGF1R/InsR levels $(\mathrm{p}=2.15 \mathrm{e}-08$; Fig $4 \mathrm{G})$ were significantly increased in the ILC tumors compared to IDC tumors. Insulin was expressed at low levels with no difference between tumor type $(\mathrm{p}=0.555$, Fig S9A). Interestingly, ILC tumors exhibited a positive correlation between both IGF1 mRNA expression (Spearman rho $=0.21 ; \mathrm{p}=0.012$, Fig 4H) and IGF2 mRNA expression (Spearman rho=0.13; $\mathrm{p}=0.14$, Fig 4I) and $\mathrm{pIGF1R/InsR} \mathrm{level,} \mathrm{despite} \mathrm{having} \mathrm{significantly}$ reduced total IGF1R expression ( $\mathrm{p}=0.0073$, Fig S9B) and similar levels of total InsR expression ( $\mathrm{p}=0.169$, Fig S9C) compared to IDC. In contrast, IDC tumors did not show a correlation suggesting that presence of IGF1/2 ligand did not necessarily activate IGF1R/ 
InsR in IDC. Strikingly, the percentage of tumors with higher than median expression (across all breast tumors) of both IGF1/2 and pIGF1R/InsR is significantly higher in ILC (IGF1=56.2\%; IGF2=43.8\%) compared to IDC (IGF1=21.3\%; IGF2=22.5\%), suggesting that IGF1/2 ligand activates IGF1R signaling in these tumors more efficiently with the loss of E-cadherin (IGF1 = chi-square test, $\mathrm{p}=2.5 \mathrm{e}-14$ [Fig 4H]; IGF2= chi-square test, $\mathrm{p}=2.5 \mathrm{e}-06$ [Fig4I]).

\section{IGF1R inhibitors and endocrine therapy synergize to decrease viability in ILC cells}

Clinically, patients with ER+ ILC are treated with endocrine therapy targeting ER, however, data from the BIG 1-98 trial suggest that ILC tumors demonstrate resistance to tamoxifen, a selective estrogen receptor modulator, compared to $\mathrm{IDC}^{30}$. Additionally, results from multiple clinical studies indicate that ILC patients have a poorer prognosis with more frequent late recurrences compared to IDC $^{31-33}$. This highlights the need to improve therapeutic options in ILC patients based on uniquely activated pathways and therefore, we evaluated efficacy of IGF1R/InsR pathway inhibitors in ER+ ILC cell lines in combination with endocrine therapy. Recent data published from our lab suggest that tamoxifen, can act as a partial ER agonist activating ER activity in some ILC cell lines, rather than a pure antagonist as in IDC cells ${ }^{34}$, in line with the data from the BIG1-98 study. Therefore, we tested efficacy of the selective estrogen receptor downregulator, ICI 182,780 (ICI) in combination with two IGF1R/InsR inhibitors used in Figure 2 (OSI and BMS) and a PI3K/ mTOR inhibitor (BEZ235 [BEZ]). SUM44PE and MM134 cells were treated with increasing doses of OSI (Fig 5A-B; Fig S10A-B), BMS (Fig 5C-D; Fig S10C-D), and BEZ (Fig 5E-F; Fig S10E-F) in combination with increasing doses of ICI. With all three IGF1R pathway inhibitors, decreased cell viability was observed with the addition of increasing doses of ICI. Formal synergy testing of the drug combinations using the Median-Effect Principle and Combination-Index Isobologram Theorem, commonly referred to as the ChouTalalay method ${ }^{27}$ revealed combination index (CI) values less than 1 for drug interactions at the ED50, ED75, and ED90 indicating a high level of synergy for the three sets of inhibitor combinations (Fig 5, Fig S10, Table S1). The lowest CI values were observed for the BMS $+\mathrm{ICI}$ drug combination in SUM44PE cells (ED50=0.127, ED75=0.081, ED90=0.099). Because the CI values from the combination of OSI or BMS with ICI in SUM44PE cells indicated the highest level of synergy in our experiments, we further tested and validated synergy using the SynergyFinder platform, with the ZIP method as output (Fig S11) ${ }^{35}$. Additionally, a minimum dose reduction index (DRI) for ICI of 8-fold for all drug combinations in SUM44PE cells and 2-fold in MM134 cells at the EC50 was seen. This data suggests that adding an IGF1R pathway inhibitor in combination with ICI reduces the concentration of ICI necessary to achieve that same inhibitory effect as ICI alone.

\section{Ex vivo IGF1R inhibition inhibits proliferation in an ILC xenograft}

Finally, we evaluated the efficacy of an IGF1R/InsR inhibitor in ILC tumors. However, there are a limited number of ILC patient-derived xenograft (PDX) and cell line xenograft models, and their slow growth rates makes large scale in vivo studies challenging. We therefore treated two ILC cell line xenografts ex vivo as explant cultures, as previously described ${ }^{36-39}$. The advantages of this technique include less tissue requirement for the assay compared to an in vivo study and rapid understanding of the therapeutic efficacy of the inhibitor. 
Additionally, data published by Majumder et al. ${ }^{39}$ suggest a high concordance between $e x$ vivo and in vivo tumor response to drug treatment. MM134 and BCK4 cells (a weakly ER responsive ILC cell line, not used for synergy experiments due to slow growth in vitro) were grown as xenografts, harvested and plated as explant culture, and treated with vehicle or BMS $(1 \mu \mathrm{M})$ for 72 hours. The tissue was collected and stained for Ki67 as a marker of proliferation. We observed a significant decrease in Ki67 positive nuclei in both tumor models treated with BMS (Fig 6). In the MM134 tumor we observed a significant decrease $(\mathrm{p}=0.002)$ in Ki67 positive nuclei from $47 \%$ in the vehicle to $22 \%$ in the BMS treated tumor tissue ( $\mathrm{n}=3$ or 4 ; Fig 6A-C). Similarly, in the BCK4 tumor we observed a significant decrease ( $\mathrm{p}=0.005$ ) in Ki67 positive nuclei from $25 \%$ in the vehicle to $11 \%$ in the BMS treated tumor tissue ( $\mathrm{n}=6$; Fig $6 \mathrm{D}-\mathrm{F})$. This data suggests that targeting IGF1R/InsR in ILC tumors may be a useful strategy to inhibit cell proliferation.

\section{DISCUSSION}

Despite a large body of preclinical evidence supporting the use of IGF1R/InsR inhibitors for the treatment of breast cancer, the outcomes of clinical trials testing the efficacy of these drugs in patients thus far have been disappointing. However, these trials proceeded with a lack of appropriate biomarkers for predicting positive therapeutic efficacy and little to no understanding of which tumor types would benefit ${ }^{13-15,40}$. In response, in recent years the field has emphasized the need to understand and identify gene expression or proteomic biomarkers that predict a positive response to targeted therapy. Along this thought process, we previously published a gene expression signature used to identify tumors that are IGF1 responsive ${ }^{16}$ and here we focus on one proteomic biomarker, E-cadherin, identified through an integrative computational approach recently published by our group ${ }^{17}$. It is known that constitutive IGF1R activation drives E-cadherin transcriptional repression through $\mathrm{EMT}^{6,10}$, however, the reverse regulation of IGF1R by E-cadherin has not been previously characterized. Our data suggest that loss of E-cadherin in breast tumors, specifically in ILC, highlights a subset of tumors that may be responsive to IGF1R inhibition and here we begin to describe the mechanism by which this regulation occurs. Data from our lab and others additionally suggest a role for IGF1/2 signaling and efficacy of IGF1R/InsR inhibition in ER-negative (particularly triple negative [TNBC]) breast tumors and cancer stem-like cells that display EMT properties (e.g. low E-cadherin expression) ${ }^{41-43}$. Therefore, our findings may potentially be extended into those settings as well.

We demonstrate that in breast cancer cells, IGF1R is endogenously localized at the cell membrane to cell-cell contacts and to intracellular compartments, potentially membranebound compartments or endosomal vesicles, similar to data published in MCF-7 cells overexpressing IGF1R ${ }^{44}$ and in corneal epithelial cells ${ }^{45}$. We show a direct, endogenous interaction between IGF1R and E-cadherin using in situ proximity ligation assay. To our knowledge interaction between IGF1R and E-cadherin in breast cancer cells has only been demonstrated by immunoprecipitation (IP) ${ }^{44}$. Our data provide confirmation of this interaction using a technique known to be higher in specificity and sensitivity compared to IP, which requires intense cell manipulation (e.g. lysis and scraping) and often results in pull-down of entire protein complexes. This suggests that IGF1R is recruited to adherens junctions by E-cadherin, possibly resulting in receptor sequestration and signaling 
repression. This process is similar to the sequestration of EGFR into the adherens junction and loss of receptor mobility, a well characterized mechanism of EGFR signaling repression ${ }^{46-48}$. However, data published by Curto et al. suggests that the latter action is mediated through the tumor suppressor, Merlin, responsible for coordinating stabilization of the adherens junction and thereby regulating contact-inhibition growth ${ }^{48}$. Although IGF1R signaling is controlled in a contact-dependent manner (Fig 1E), they also showed that IGF1 activity is not regulated by Merlin, indicating that IGF1R regulation by E-cadherin likely occurs independent of this factor ${ }^{48}$. Although there may be a yet undefined intermediate regulator similar to Merlin, our data indicate that E-cadherin plays a role in coordinating the recruitment and sequestration of IGF1R within the adherens junction to repress IGF1R signaling. When E-cadherin is lost and junction formation is disrupted (such as in ILC cells), IGF1R is released and re-localizes to the entirety of the cell membrane where signaling is more easily initiated upon IGF1 ligand binding.

Additionally, E-cadherin may potentially regulate IGF1R trafficking and/or endocytosis as a mechanism of controlling maximal signaling capacity, potentially an explanation for the protein complex localization to non-cell membranous compartments of the cell. Both IGF1R and E-cadherin cell surface expression is known to be regulated by clathrin-mediated endocytosis, and Romanelli et al. described the requirement for IGF1R internalization via endocytosis for maximal activation of IGF1-induced Akt signaling, showing that inhibition of IGF1R internalization was sufficient to block maximal Akt activation ${ }^{49-53}$. Based on this, we hypothesize that E-cadherin presence at the cell membrane potentially regulates IGF1R endocytosis as a mechanism of normal growth factor signaling control. However, the loss of E-cadherin, such as with siRNA knockdown, may allow for unchecked IGF1R internalization and enhanced signaling capacity. A similar mechanism of regulation has been described by Bryant et al. such that FGF-induced activation of FGFR1 receptor resulted in co-internalization of FGFR1 and E-cadherin and subsequent translocation of FGFR1 to the nucleus. Importantly, the overexpression of E-cadherin reduced FGFR1 internalization and therefore, disrupted maximal FGFR1 signaling ${ }^{49,53}$. This hypothesis warrants further investigation to fully understand the mechanism of action of the IGF1R/E-cadherin complex on IGF1R signaling regulation. Initial attempts to investigate IGF1R internalization and trafficking using IGF1R surface biotinylation or cellular fractionation experiments have not yet been successful due to current technical limitations and the scope of this study.

Supporting this concept, our data indicate that the knockdown of E-cadherin in three ER+ breast cancer cell lines not only enhanced IGF1-induced signaling via IGF1R/InsR but also increased sensitivity of the cells to the ligand. This is similar to the relationship reported between EGF-EGFR and IGF1-IGF1R upon adherens junction disruption via calciumdepletion ${ }^{46}$. Because of the increased IGF1R pathway activation associated with the loss of E-cadherin, the knockdown cells in turn became more sensitive to IGF1R/InsR inhibition. Future studies will serve to investigate the effect of E-cadherin overexpression on IGF1R signaling in the E-cadherin deficient ILC breast cancer cell lines.

We believe that IGF1R signaling may be particularly important in ILC, an understudied subtype of breast cancer, due to the complete loss of E-cadherin protein and/or adherens junction formation. In this subtype, the loss of E-cadherin may serve as a biomarker of IGF1 
activity. Indeed, we demonstrate that ILC have increased IGF1R pathway activation (IGF1 ligand expression and pIGF1R/InsR levels) compared with IDC. This is similar to the results of two studies analyzing differences between ILC and IDC that found increased IGF1 ligand and IGF1R expression levels in ILC ${ }^{54,55}$. Consistent with this, we found that ILC cell lines are susceptible to IGF1R/InsR inhibition and importantly, that IGF1R pathway inhibitors (OSI, BMS, BEZ) synergize with a standard of care endocrine therapy (ICI) resulting in further reduced cell growth. Future studies will focus on validating these therapies in additional ILC tumors and understanding the synergistic interaction between IGF1R/InsR inhibitors and ICI, and additionally determining if re-expression of E-cadherin inhibits IGF1R/InsR inhibition. This data may be especially important given that there is an increased prevalence of late recurrences in ER+ ILC compared to ER+ IDC tumors treated with endocrine therapy, indicating the need for improved therapy options for patients with ILC $^{30,31}$.

One limitation for the use of IGF1R inhibitors in ILC is the relatively high prevalence of mutations in the PI3K/Akt signaling pathway. Recently, Ciriello et al. comprehensively characterized ILC tumors compared to IDC tumors and described the mutational landscape of 127 ILC tumors ${ }^{23}$. They found that $48 \%$ of ILC harbor hotspot/missense mutations in PIK3CA and $13 \%$ have alterations in PTEN, similar to previously published data ${ }^{25,45}$. These genetic alterations likely lead to the elevated Akt signature they reported in these tumors. Our data suggest that the remaining tumors may also have high PI3K/Akt signaling activity due to aberrant IGF1R activity. But, because the alterations in PIK3CA/PTEN occur downstream of IGF1R, the effectiveness of IGF1R inhibition in this setting is unclear. Resistance to other upstream kinase inhibitors in tumors harboring activating alterations in PIK3CA/PTEN has been previously observed ${ }^{57}$ and therefore, it would be important to screen patients for these alterations before considering use of receptor tyrosine kinase inhibitor therapy. However, the use of a PI3K pathway inhibitor, such as BEZ235, may be mutually beneficial in targeting the PIK3CA/PTEN alterations and the enhanced IGF1R pathway activation observed in these tumors. Interestingly, Cantley et al. recently reported that high levels of insulin promote resistance to PI3K inhibitors in tumors with PIK3CA mutations ${ }^{58}$, and therefore there may also be a role for combinatorial IGF1R and PI3K inhibition. Future studies are warranted to investigate these relationships using additional $e x$ vivo or in vivo screening of ILC tumors.

In summary, we present a diverse set of data indicating that the loss of E-cadherin enhances IGF1R pathway activity and sensitivity to IGF1R/InsR therapy, specifically in ILC. We show that IGF1R and E-cadherin directly interact, which potentially leads to the sequestration and potential repression of IGF1R within the adherens junction. Overall, this study begins to shed light on a previously unrecognized mechanism of IGF1R regulation by E-cadherin and highlights a potential therapeutic strategy of exploiting IGF1R pathway activity in ILC tumors.

\section{Supplementary Material}

Refer to Web version on PubMed Central for supplementary material. 


\section{ACKNOWLEDGEMENTS}

Additional funding and support was provided by UPMC Hillman Cancer Center/Women's Cancer Research Center. AVL and SO are recipients of Scientific Advisory Council awards from Susan G. Komen for the Cure, and AVL is a Hillman Foundation Fellow. The authors acknowledge Drs. Damir Vareslija and Leone Young for providing their expertise and media conditions for use in tumor explant culturing.

Financial support:

This study was supported in part by The Breast Cancer Research Foundation (AVL), NIH/NCI R01CA94118 (AVL), NIH/NCI F31 CA224567 (AMN), NIH T32 GM008424 (AMN), NIH/NCI F30 CA203154 (KML), and Department of Defense Breakthrough Fellowship Award BC160764 (NT).

\section{REFERENCES}

1. Pollak MN Insulin-like growth factors and neoplasia. Nat. Rev. Cancer 262, 84-107,265-268 (2004).

2. Becker M. a, Ibrahim YH, Cui X, Lee AV \& Yee D The IGF pathway regulates ERa through a S6K1-dependent mechanism in breast cancer cells. Mol. Endocrinol 25, 516-28 (2011). [PubMed: 21292829]

3. Hawsawi Y, El-Gendy R, Twelves C, Speirs V \& Beattie J Insulin-like growth factor - oestradiol crosstalk and mammary gland tumourigenesis. Biochim. Biophys. Acta 1836, 345-53 (2013). [PubMed: 24189571]

4. Yee D \& Lee AV Crosstalk between the insulin-like growth factors and estrogens in breast cancer. J. Mammary Gland Biol. Neoplasia 5, 107-15 (2000). [PubMed: 10791773]

5. Baserga R, Peruzzi F \& Reiss K The IGF-1 receptor in cancer biology. Int. J. Cancer 107, 873-877 (2003). [PubMed: 14601044]

6. Kim H-J et al. Constitutively active type I insulin-like growth factor receptor causes transformation and xenograft growth of immortalized mammary epithelial cells and is accompanied by an epithelial-to-mesenchymal transition mediated by NF-kappaB and snail. Mol. Cell. Biol 27, 316575 (2007). [PubMed: 17296734]

7. Litzenburger BC et al. BMS-536924 Reverses IGF-IR-induced Transformation of Mammary Epithelial Cells and Causes Growth Inhibition and Polarization of MCF7 Cells. Clin Cancer Res 15, 1-23 (2009). [PubMed: 19118025]

8. Carboni JM et al. Tumor Development by Transgenic Expression of a Constitutively Active InsulinLike Growth Factor I Receptor. 3781-3788 (2005).

9. Sachdev D, Hartell JS, Lee AV, Zhang X \& Yee D A Dominant Negative Type I Insulin-like Growth Factor Receptor Inhibits Metastasis of Human Cancer Cells. J. Biol. Chem 279, 5017-5024 (2004). [PubMed: 14615489]

10. Cox OT et al. IGF-1 Receptor and Adhesion Signaling: An Important Axis in Determining Cancer Cell Phenotype and Therapy Resistance. Front. Endocrinol. (Lausanne). 6, 106 (2015). [PubMed: 26191041]

11. Farabaugh SM, Boone DN \& Lee AV Role of IGF1R in Breast Cancer Subtypes, Stemness, and Lineage Differentiation. Front. Endocrinol. (Lausanne). 6, 59 (2015). [PubMed: 25964777]

12. Crudden C, Girnita A \& Girnita L Targeting the IGF-1R: The Tale of the Tortoise and the Hare. Front. Endocrinol. (Lausanne). 6, 64 (2015). [PubMed: 25964779]

13. Boone DN \& Lee AV Targeting the Insulin-like Growth Factor Receptor: Developing Biomarkers from Gene Expression Profiling. Crit Rev Oncog 17, 161-173 (2012). [PubMed: 22471706]

14. Singh P, Alex JM \& Bast F Insulin receptor (IR) and insulin-like growth factor receptor 1 (IGF-1R) signaling systems: novel treatment strategies for cancer. Med. Oncol 31, 805 (2014). [PubMed: 24338270]

15. Ekyalongo RC \& Yee D Revisiting the IGF-1R as a breast cancer target. npj Precis. Oncol 1, 14 (2017). [PubMed: 29152592] 
16. Creighton CJ et al. Insulin-like growth factor-I activates gene transcription programs strongly associated with poor breast cancer prognosis. J. Clin. Oncol 26, 4078-85 (2008). [PubMed: 18757322]

17. Erdem C et al. Proteomic Screening and Lasso Regression Reveal Differential Signaling in Insulin and Insulin-like Growth Factor I (IGF1) Pathways. Mol. Cell. Proteomics 15, 3045-3057 (2016). [PubMed: 27364358]

18. Onder TT et al. Loss of E-cadherin promotes metastasis via multiple downstream transcriptional pathways. Cancer Res. 68, 3645-3654 (2008). [PubMed: 18483246]

19. Hanahan D \& Weinberg RA Hallmarks of cancer: The next generation. Cell 144, 646-674 (2011). [PubMed: 21376230]

20. Mauro L et al. Role of the IGF-I receptor in the regulation of cell-cell adhesion: Implications in cancer development and progression. J. Cell. Physiol 194, 108-116 (2003). [PubMed: 12494449]

21. Friedl P \& Alexander S Cancer invasion and the microenvironment: Plasticity and reciprocity. Cell 147, 992-1009 (2011). [PubMed: 22118458]

22. Roxanis I Occurrence and significance of epithelial-mesenchymal transition in breast cancer. J. Clin. Pathol 66, 517-21 (2013). [PubMed: 23322823]

23. Ciriello G et al. Comprehensive Molecular Portraits of Invasive Lobular Breast Cancer. Cell 163, 506-519 (2015). [PubMed: 26451490]

24. Barroso-Sousa R \& Metzger-Filho O Differences between invasive lobular and invasive ductal carcinoma of the breast: results and therapeutic implications. Ther. Adv. Med. Oncol 8, 261-266 (2016). [PubMed: 27482285]

25. Rakha EA et al. Clinical and biological significance of E-cadherin protein expression in invasive lobular carcinoma of the breast. Am J Surg Pathol 34, 1472-1479 (2010). [PubMed: 20871222]

26. Jambal $P$ et al. Estrogen switches pure mucinous breast cancer to invasive lobular carcinoma with mucinous features. Breast Cancer Res. Treat 137, 431-448 (2013). [PubMed: 23247610]

27. Chou TC \& Talalay P Quantitative analysis of dose-effect relationships: the combined effects of multiple drugs or enzyme inhibitors. Adv. Enzyme Regul 22, 27-55 (1984). [PubMed: 6382953]

28. Marcotte R et al. Functional Genomic Landscape of Human Breast Cancer Drivers, Vulnerabilities, and Resistance. Cell 164, 293-309 (2016). [PubMed: 26771497]

29. Derksen PWB et al. Somatic inactivation of E-cadherin and p53 in mice leads to metastatic lobular mammary carcinoma through induction of anoikis resistance and angiogenesis. Cancer Cell 10, 437-449 (2006). [PubMed: 17097565]

30. Filho $\mathrm{OM}$ et al. Relative effectiveness of letrozole compared with tamoxifen for patients with lobular carcinoma in the BIG 1-98 Trial. J. Clin. Oncol 33, 2772-2778 (2015). [PubMed: 26215945]

31. Pestalozzi BC et al. Distinct clinical and prognostic features of infiltrating lobular carcinoma of the breast: Combined results of 15 International Breast Cancer Study Group clinical trials. J. Clin. Oncol 26, 3006-3014 (2008). [PubMed: 18458044]

32. Chen $\mathrm{Z}$ et al. Invasive lobular carcinoma of the breast: A special histological type compared with invasive ductal carcinoma. PLoS One 12, 1-17 (2017).

33. Adachi $\mathrm{Y}$ et al. Comparison of clinical outcomes between luminal invasive ductal carcinoma and luminal invasive lobular carcinoma. BMC Cancer 16, 1-9 (2016).

34. Sikora $\mathrm{MJ}$ et al. Invasive lobular carcinoma cell lines are characterized by unique estrogenmediated gene expression patterns and altered tamoxifen response. Cancer Res. 74, 1463-1474 (2014). [PubMed: 24425047]

35. Ianevski A, He L, Aittokallio T \& Tang J SynergyFinder: A web application for analyzing drug combination dose-response matrix data. Bioinformatics 33, 2413-2415 (2017). [PubMed: 28379339]

36. Andersen CL et al. Active estrogen receptor-alpha signaling in ovarian cancer models and clinical specimens. Clin. Cancer Res. clincanres.1501.2016 (2017). doi:10.1158/1078-0432.CCR-16-1501

37. Centenera MM, Raj GV, Knudsen KE, Tilley WD \& Butler LM Ex vivo culture of human prostate tissue and drug development. Nat. Rev. Urol 10, 483-487 (2013). [PubMed: 23752995] 
38. Dean JL et al. Therapeutic response to CDK4/6 inhibition in breast cancer defined by ex vivo analyses of human tumors. Cell Cycle 11, 2756-2761 (2012). [PubMed: 22767154]

39. Majumder B et al. Predicting clinical response to anticancer drugs using an ex vivo platform that captures tumour heterogeneity. Nat. Commun 6, 6169 (2015). [PubMed: 25721094]

40. Gualberto a \& Pollak M Emerging role of insulin-like growth factor receptor inhibitors in oncology: early clinical trial results and future directions. Oncogene 28, 3009-3021 (2009). [PubMed: 19581933]

41. Tominaga $\mathrm{K}$ et al. Addiction to the IGF2-ID1-IGF2 circuit for maintenance of the breast cancer stem-like cells. Oncogene 1-11 (2016). doi:10.1038/onc.2016.293

42. Litzenburger BC et al. High IGF-IR activity in triple-negative breast cancer cell lines and tumorgrafts correlates with sensitivity to anti-IGF-IR therapy. Clin. Cancer Res 17, 2314-27 (2011). [PubMed: 21177763]

43. Ireland $\mathrm{L}$ et al. Blockade of insulin-like growth factors increases efficacy of paclitaxel in metastatic breast cancer. Oncogene 1 (2018). doi:10.1038/s41388-017-0115-x

44. Guvakova M. a \& Surmacz E Overexpressed IGF-I receptors reduce estrogen growth requirements, enhance survival, and promote E-cadherin-mediated cell-cell adhesion in human breast cancer cells. Exp. Cell Res 231, 149-62 (1997). [PubMed: 9056422]

45. Robertson DM, Zhu M \& Wu Y-C Cellular distribution of the IGF-1R in corneal epithelial cells. Exp. Eye Res 94, 179-86 (2012). [PubMed: 22193032]

46. Qian X, Karpova T, Sheppard AM, McNally J \& Lowy DR E-cadherin-mediated adhesion inhibits ligand-dependent activation of diverse receptor tyrosine kinases. EMBO J. 23, 1739-1784 (2004). [PubMed: 15057284]

47. de-Freitas-Junior JCM et al. Insulin/IGF-I Signaling Pathways Enhances Tumor Cell Invasion through Bisecting GlcNAc N-glycans Modulation. An Interplay with E-Cadherin. PLoS One 8, e81579 (2013). [PubMed: 24282611]

48. Curto M, Cole BK, Lallemand D, Liu CH \& McClatchey AI Contact-dependent inhibition of EGFR signaling by Nf2/Merlin. J. Cell Biol 177, 893-903 (2007). [PubMed: 17548515]

49. Cadwell CM, Su W \& Kowalczyk AP Cadherin tales: Regulation of cadherin function by endocytic membrane trafficking. Traffic 17, 1262-1271 (2016). [PubMed: 27624909]

50. Brüser L \& Bogdan S Adherens Junctions on the Move-Membrane Trafficking of E-Cadherin. Cold Spring Harb. Perspect. Biol 9, a029140 (2017). [PubMed: 28096264]

51. Malaguarnera R et al. Novel cross talk between IGF-IR and DDR1 regulates IGF-IR trafficking, signaling and biological responses. Oncotarget 6, (2015).

52. Romanelli RJ et al. Insulin-like growth factor type-I receptor internalization and recycling mediate the sustained phosphorylation of Akt. J. Biol. Chem 282, 22513-22524 (2007). [PubMed: 17545147]

53. Bryant DM, Wylie FG \& Stow JL Regulation of Endocytosis, Nuclear Translocation, and Signaling of Fibroblast Growth Factor Receptor 1 by E-Cadherin. Mol. Biol. Cell 16, 14-23 (2005). [PubMed: 15509650]

54. Nakagawa $S$ et al. Tumor microenvironment in invasive lobular carcinoma: possible therapeutic targets. Breast Cancer Res. Treat 155, 65-75 (2016). [PubMed: 26715212]

55. Bertucci $\mathrm{F}$ et al. Lobular and ductal carcinomas of the breast have distinct genomic and expression profiles. Oncogene 27, 5359-5372 (2008). [PubMed: 18490921]

56. Shah V et al. PIK3CA mutations are common in lobular carcinoma in situ, but are not a biomarker of progression. Breast Cancer Res. 19, 7 (2017). [PubMed: 28095868]

57. Wang L et al. PI3K pathway activation results in low efficacy of both trastuzumab and lapatinib. BMC Cancer 11, 11:248 (2011). [PubMed: 21223585]

58. Cantley Lewis. PI 3-kinase links obesity, insulin resistance, and cancer. in (AACR-NCI-EORTC Molecular Targets and Cancer Therapeutics Meeting, 2017). 


\section{STATEMENT OF SIGNIFICANCE}

IGF1R signaling is an attractive therapeutic target in breast cancer due to its regulation of proliferation, migration, and invasion. However, clinical trials targeting IGF1R have largely been unsuccessful due to lack of biomarkers to stratify patients for therapeutic response. In this study, we demonstrate loss of E-cadherin as a potential biomarker for response to anti-IGF1R/InsR therapy, and show efficacy of IGF1R/InsR inhibition in ER + ILC in combination with endocrine therapy. Patients with ER+ ILC have poorer longterm outcomes than patients with ER+ IDC and have a propensity for increased late recurrences, highlighting the need for improved therapeutic strategies for this subtype of breast cancer. Here, we credential IGF1R/InsR inhibition as a novel therapeutic strategy in combination with endocrine therapy for the treatment of ER+ ILC. 


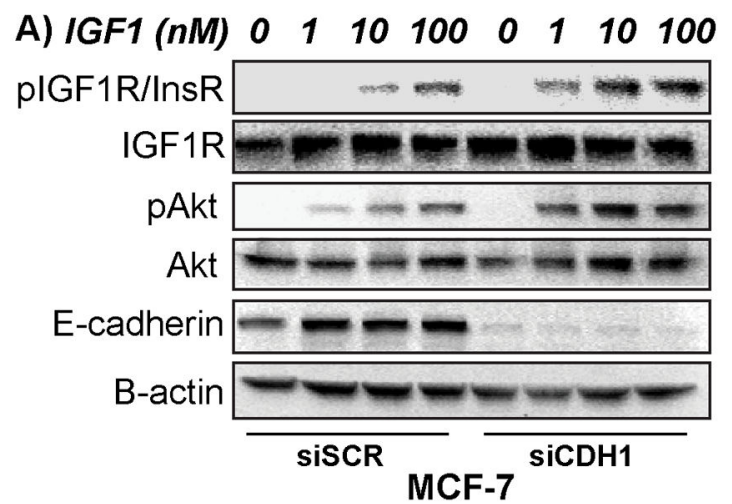

D) IGF1 (nM)
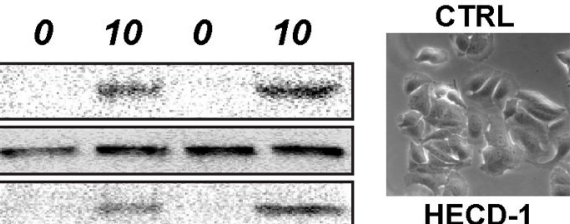

plGF1R/InsR

IGF1R

pAkt

Akt

B-actin

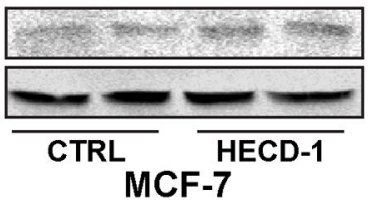

HECD-1

MCF-7

E)

IGF1

B) IGF1 (nM) $0 \quad 1 \quad 10100 \quad 0 \quad 1 \quad 10100$ plGF1R/InsR

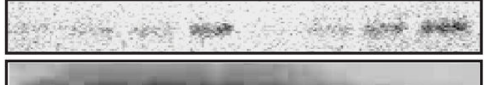

plGF1R/InsR

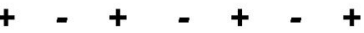

IGF1R

pAkt

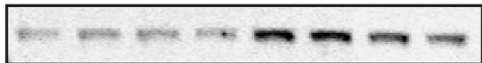

Akt

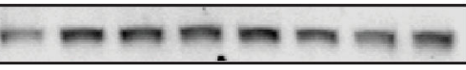

E-cadherin

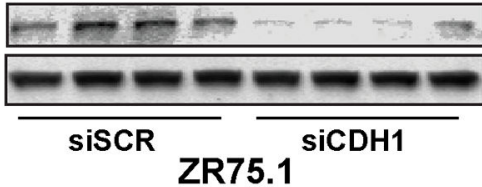

B-actin

ZR75.1

C) IGF1 (nM) $0 \quad 1 \quad 10100 \quad 0 \quad 1 \quad 10100$

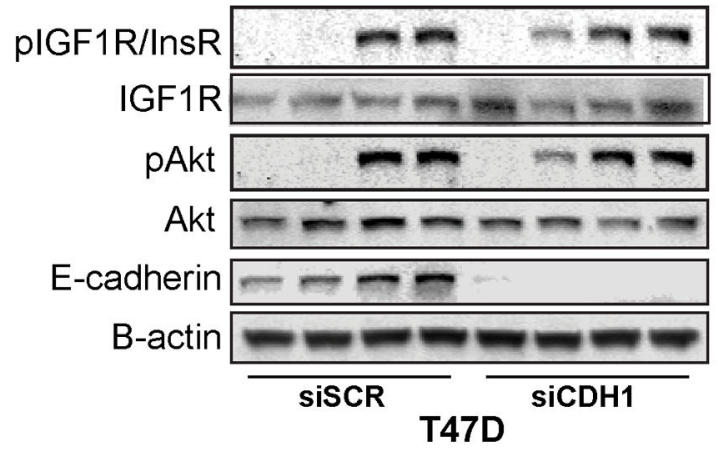

F)

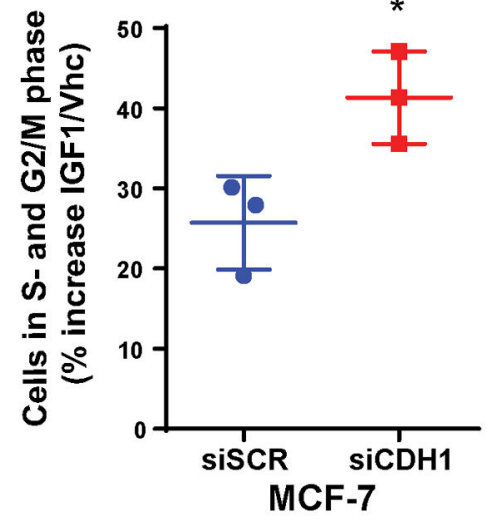

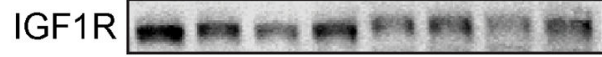

E-cadherin $=-\quad=-$

B-actin
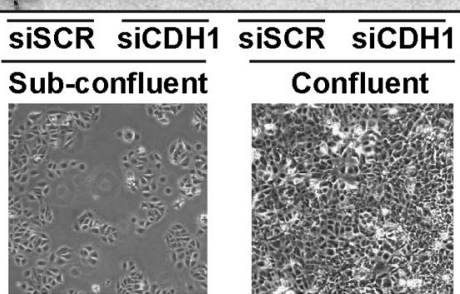

Figure 1: Loss or inhibition of E-cadherin $(\mathrm{CDH1})$ expression enhances IGF1R/InsR signaling. (A) MCF-7, (B) ZR75.1, and (C) T47D breast cancer cells transfected with SCR (siSCR) or CDH1 (siCDH1) siRNA were stimulated with increasing doses of IGF1 (0-100nM) for 10 min. IGF1R/InsR and Akt signaling was assessed by immunoblot. (D) MCF-7 cells were treated with $25 \mathrm{ug} / \mathrm{ml} \mathrm{HECD}-1$ antibody for 24 hours and imaged by phase-contrast microscopy for dissociation of adherens junctions. Cells were stimulated with Vhc or 10nM IGF1 for 10 min and IGF1R and Akt signaling assessed by immunoblot. (E) MCF-7 cells were plated at sub-confluency (200k cells in 6-well) or high confluency (800k cells) and then stimulated with either Vhc or 10nM IGF1 for $10 \mathrm{~min}$. IGF1R/InsR signaling was assessed by immunoblot. Representative phase-contrast microscopy images of the cell plating densities are shown. (F) MCF-7 and ZR75.1 siSCR and siCDH1 cells were serum- 
starved and stimulated with 10nM IGF1 for 17 hours and DNA stained with propidium iodide to measure cell cycle profile. The percent of cells in the IGF1/Vhc conditions in the S- and G2/M phases of the cell cycle for siSCR and siCDH1 are shown (representative experiment shown; $\mathrm{n}=2$ or 3 each with 3 biological replicates). 
OSI-906

BMS-754807
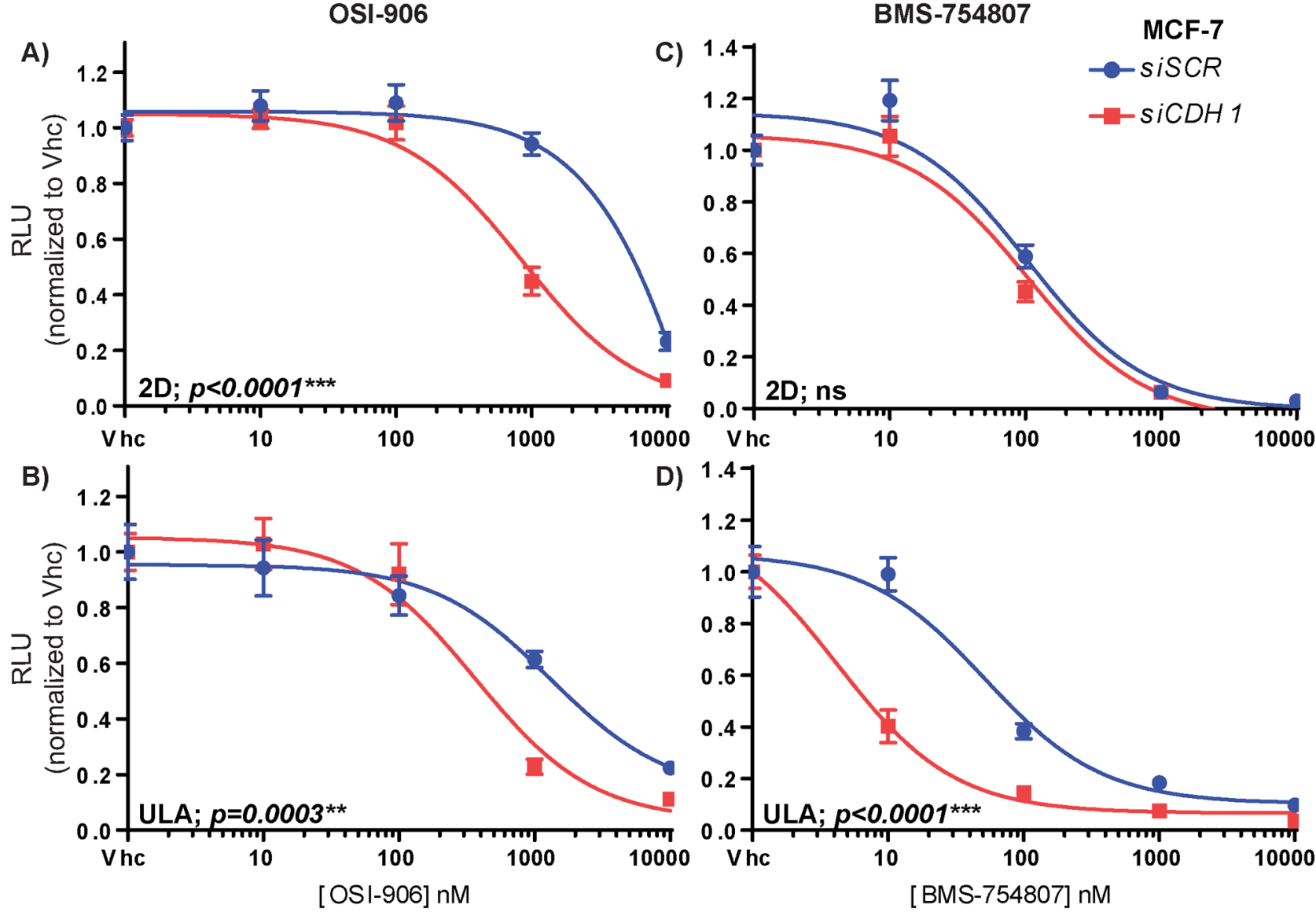

D) 1.4

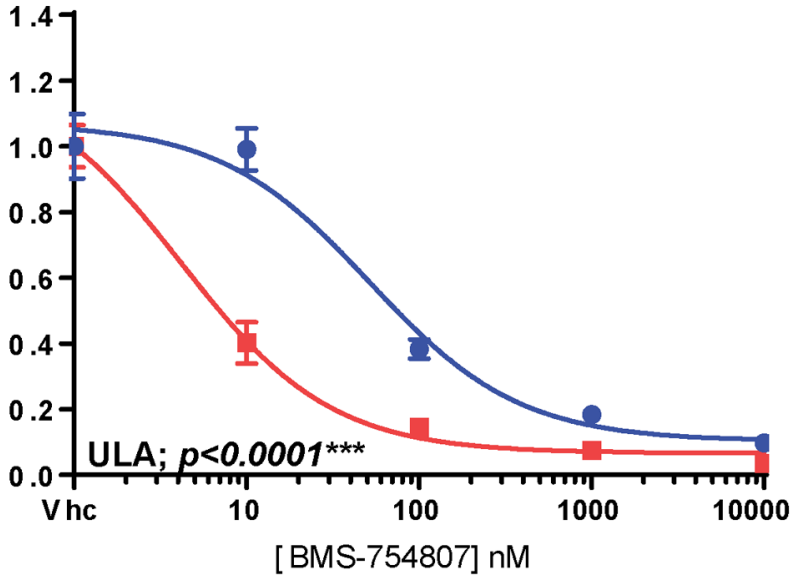

Figure 2: Knockdown of E-cadherin increases sensitivity to IGF1R/InsR inhibition in breast cancer cells.

MCF-7 cells were reverse transfected with SCR or CDH1 siRNA and seeded into 96-well 2D or ULA plates and treated with IGF1R inhibitor (OSI-906 or BMS-754807) for 6 days. Conditions in the panels as follows: (A) OSI-906; 2D, (B) OSI-906; ULA, (C) BMS-754807; 2D, (D) BMS-754807; ULA. The CellTiter Glo assay was used to assess cell viability (relative luminescence). EC50 values for viability were calculated by non-linear regression and statistical differences evaluated using sum-of-squares Global $\mathrm{f}$-test ( $\mathrm{p}<0.05$; representative experiment shown; $\mathrm{n}=3$ each with 6 biological replicates). 

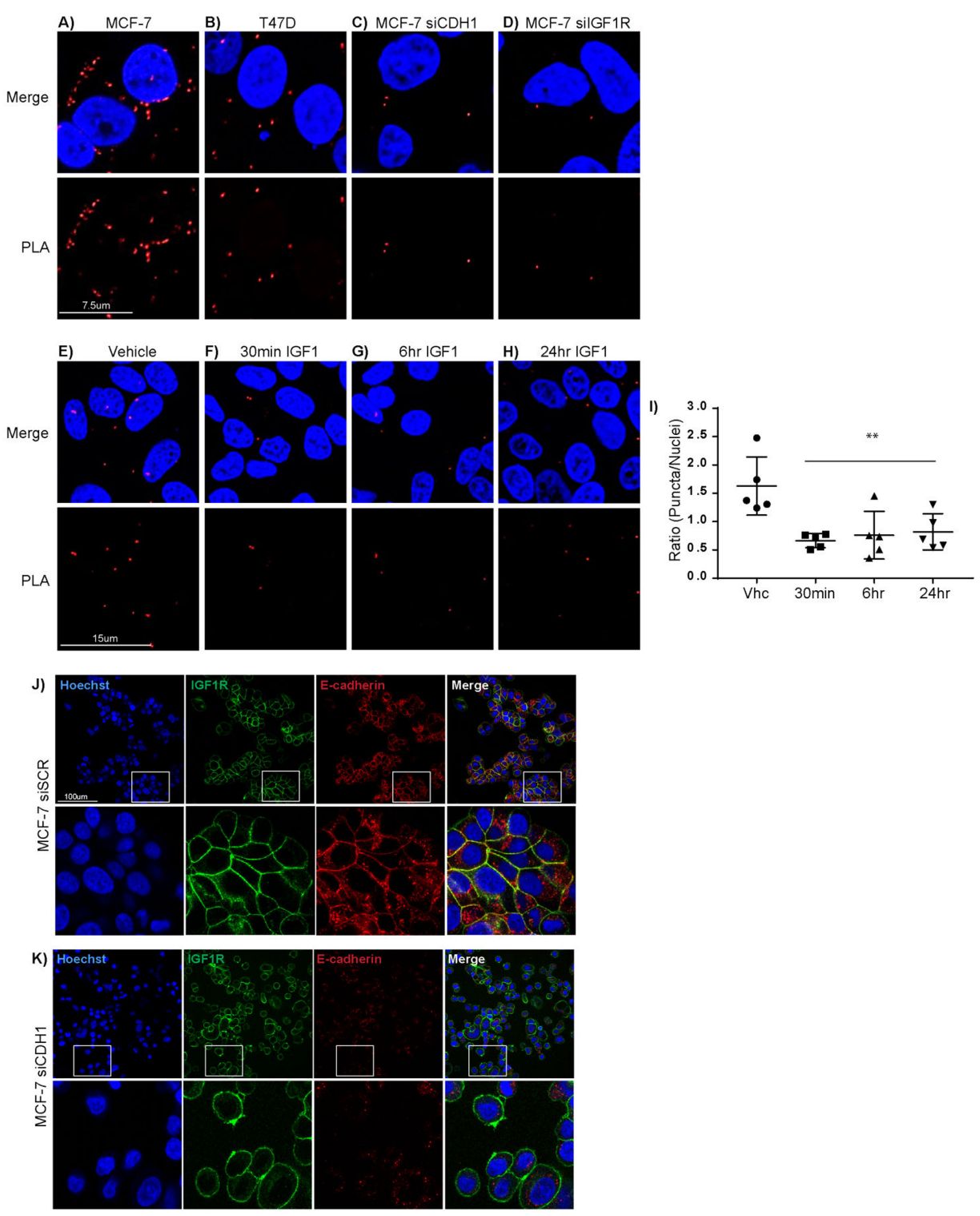

Figure 3: Proximity ligation assay reveals interaction between IGF1R and E-cadherin and recruitment of IG1R to adherens junctions.

In situ proximity ligation assay (PLA) was used to analyze the direct interaction between IGF1R and E-cadherin in breast cancer cells. (A) MCF-7 and (B) T47D cells were plated on coverslips, fixed, and stained with IGF1R and E-cadherin antibody overnight. The Duolink (Sigma) protocol was followed and coverslips were imaged using confocal microscopy to reveal red puncta. (C) MCF-7 siCDH1 and (D) siIGF1R cells were used as negative controls for the assay to assess primary antibody specificity. MCF-7 cells were plated on coverslips and treated with either (E) Vhc or 10nM IGF1 for (F) 30 minutes, (G) 6 hours, or (H) 24 hours. PLA protocol for IGF1R and E-cadherin was followed as described above. (I) Red puncta and nuclei (stained with DAPI) were quantified and displayed as a ratio of puncta/ nuclei. All puncta and nuclei in 60x images were counted. One-way ANOVA was used to determine significant difference between groups $(\mathrm{p}<0.05$; one independent experiment, $\mathrm{n}=5$ images per slide counted). The co-localization of IGF1R (green) and E-cadherin (red) was 
analyzed by immunofluorescence staining in (J) MCF-7 siSCR and (K) siCDH1 knockdown cells. 

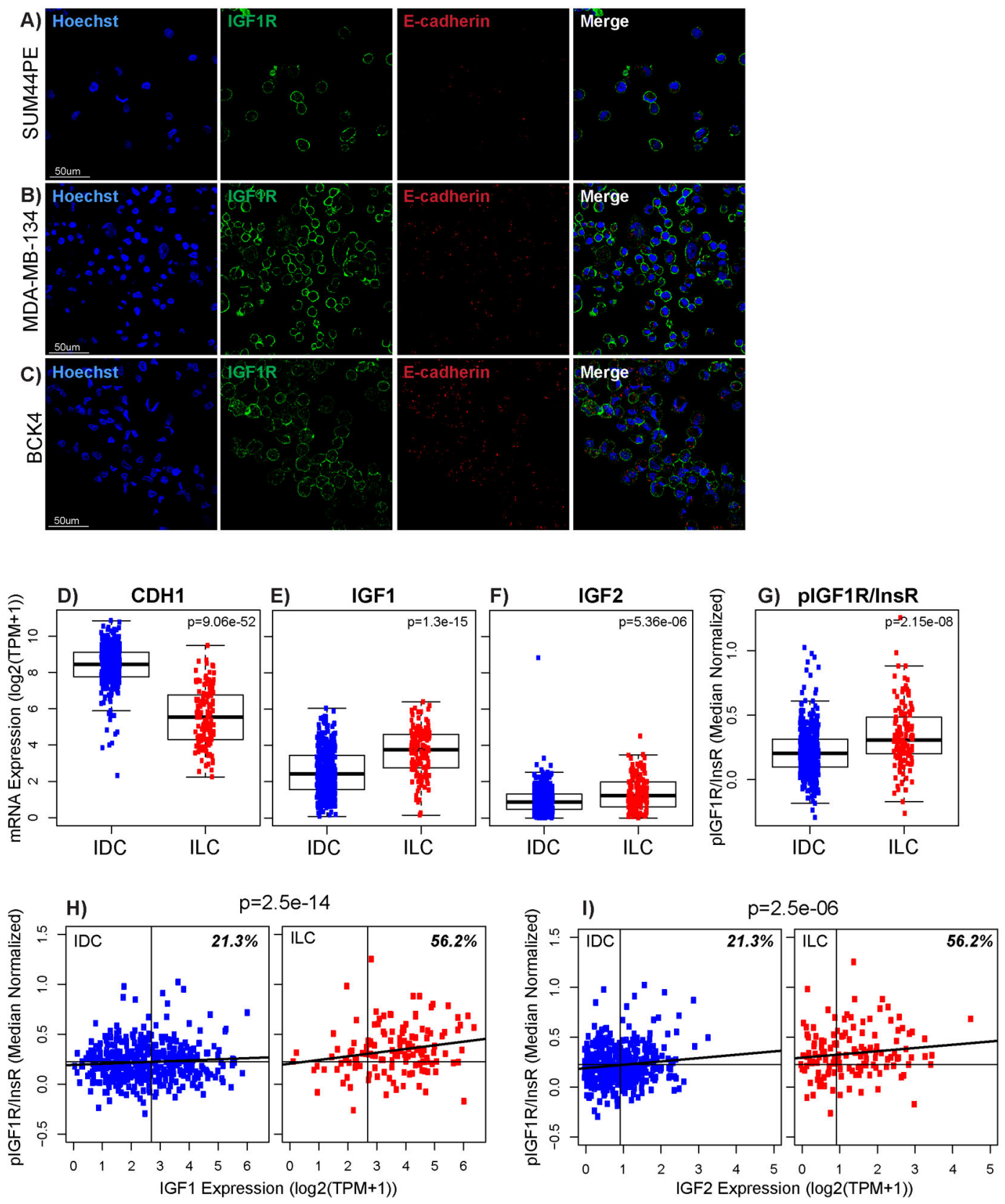

Figure 4: IGF1-IGF1R pathway is active in invasive lobular breast carcinoma with genetic loss of CDH1.

(A) SUM44PE, (B) MDA-MB-134, and (C) BCK4 ILC cells were immunostained for IGF1R (green) and E-cadherin (red) and imaged by confocal microscopy. Of note, BCK4 cells were imaged at an increased exposure compared to MM134 and SUM44PE cells. (D) CDH1 mRNA, (E) IGF1 mRNA, (F) IGF2 mRNA, and (G) pIGF1R/InsR levels in ER+ IDC compared to ER+ ILC in TCGA were plotted using RNAseq $(\log 2 \mathrm{TPM}+1)$ and RPPA (median normalized) data. The TCGA cohort includes $n=417$ IDC cases and $n=137$ ILC cases that have matched data for RNAseq and RPPA. Man-Whitney test was used to determine significant differences in expression level between the two subtypes, $\mathrm{p}<0.05$. 
Correlation between pIGF1R/InsR and (H) IGF1 and (I) IGF2 ligand expression is plotted for IDC (left) and ILC (right). 

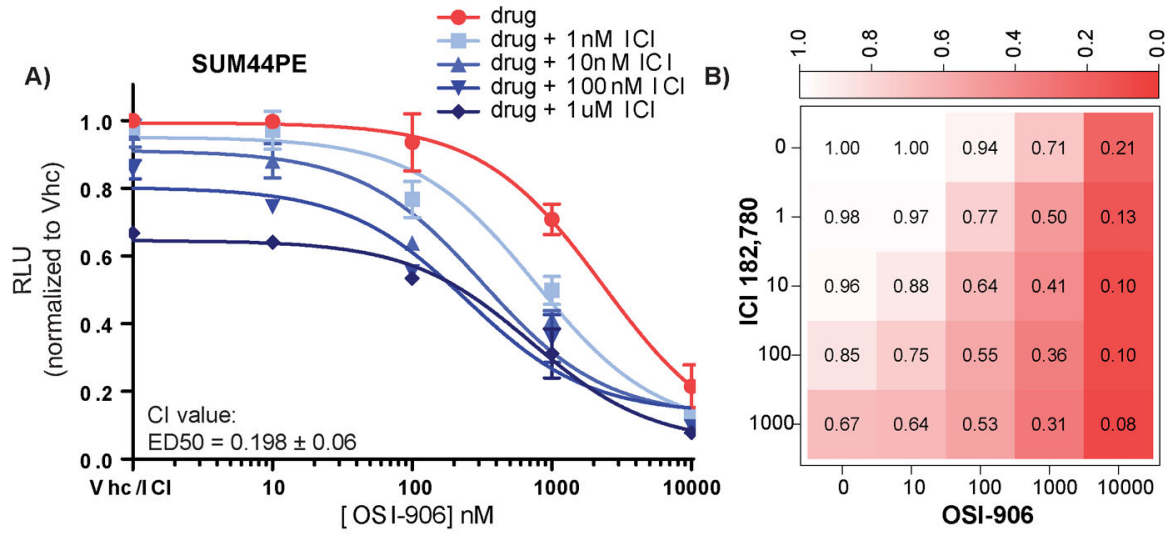

C)

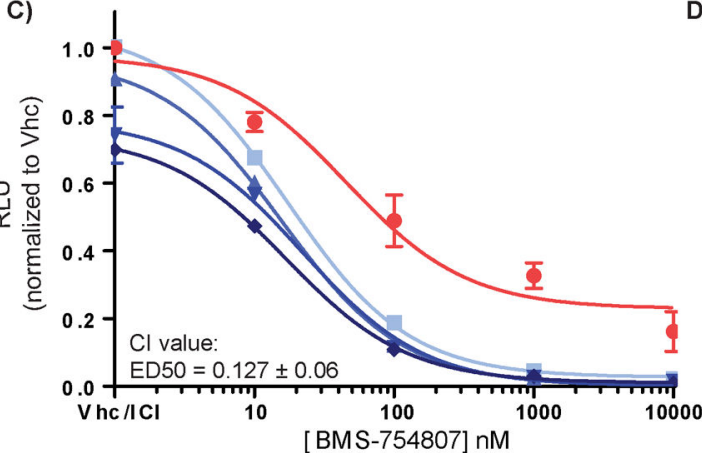

E)

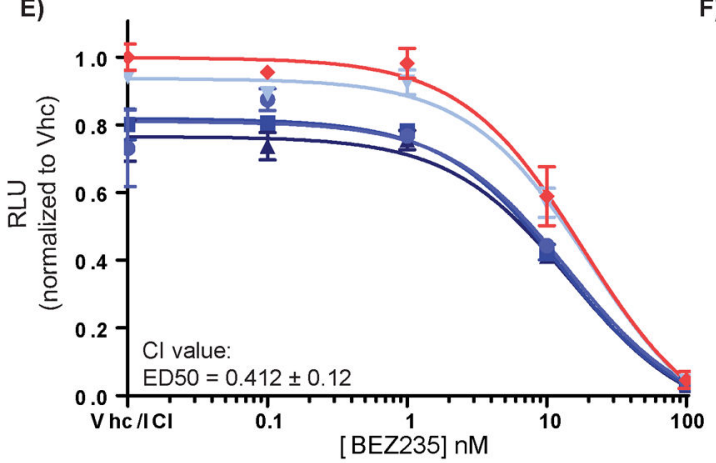

D)

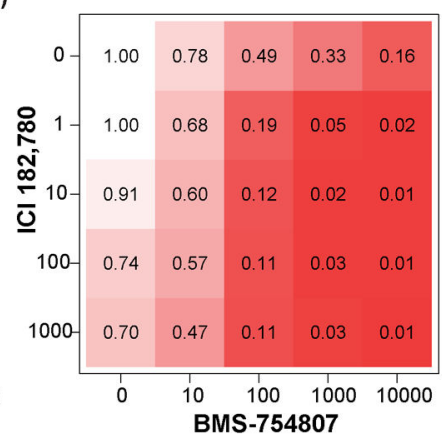

F)

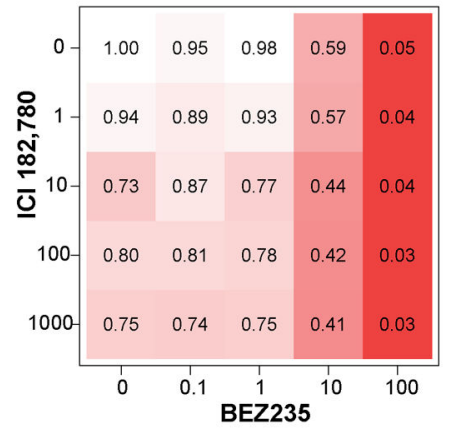

Figure 5: IGF1R pathway inhibitors and endocrine therapy synergize to inhibit cell viability in ILC breast cancer cells.

SUM44PE ILC cells were plated into 96-well ULA plates and treated for 6 days with increasing doses of (A, B) OSI-906, (C, D) BMS-754807, or (E, F) BEZ235 in combination with increasing doses of ICI 182,780. The dose response curves and heat maps shown indicate inhibition of cell viability (CellTiter Glo). Representative experiment shown; $n=3$ independent experiments each with 2 biological replicates per combination of doses. 

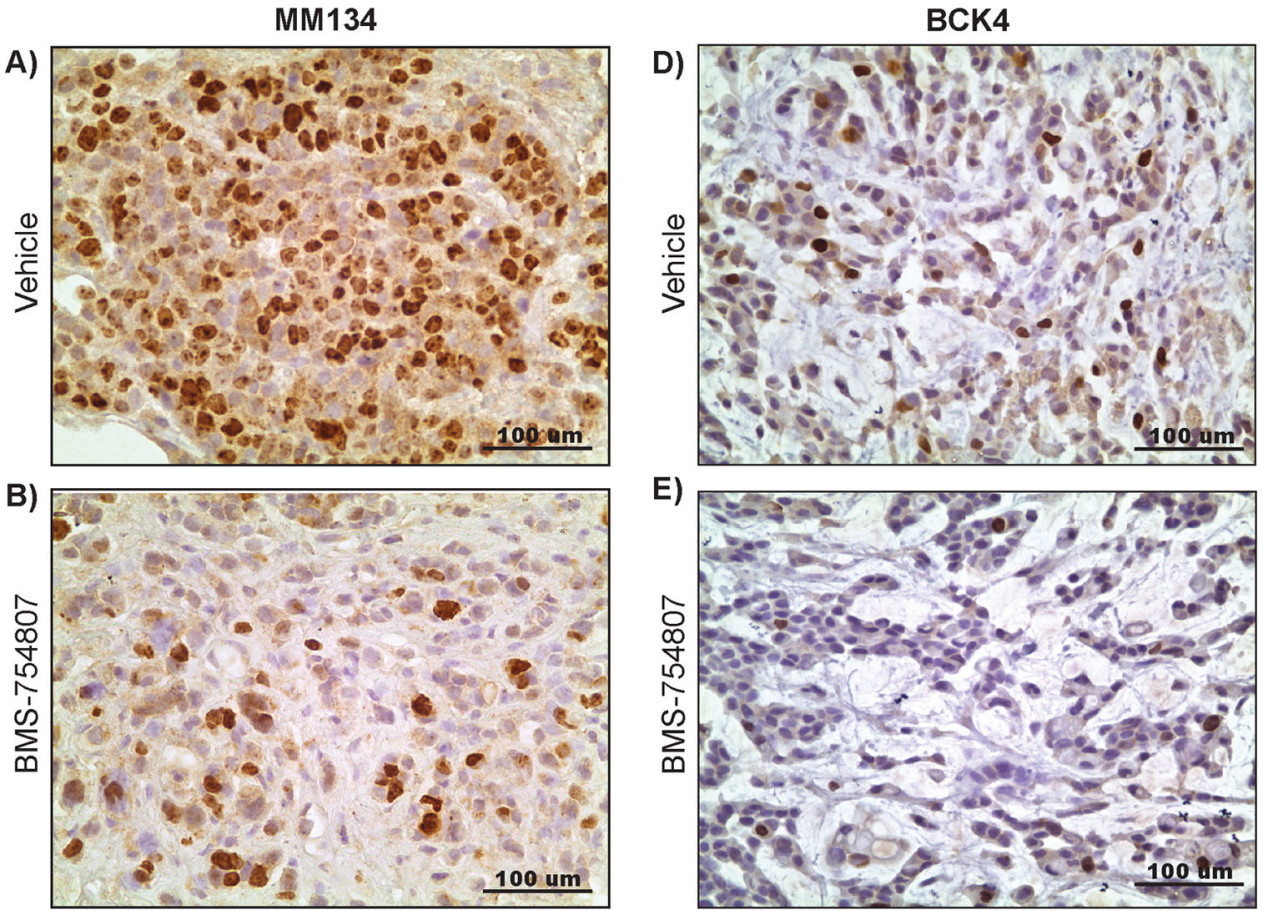

C)

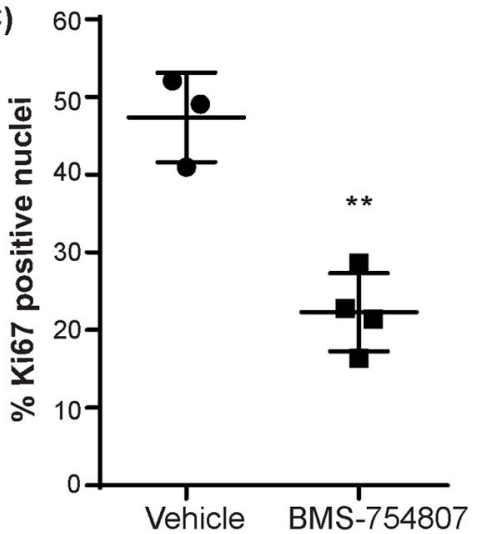

E)

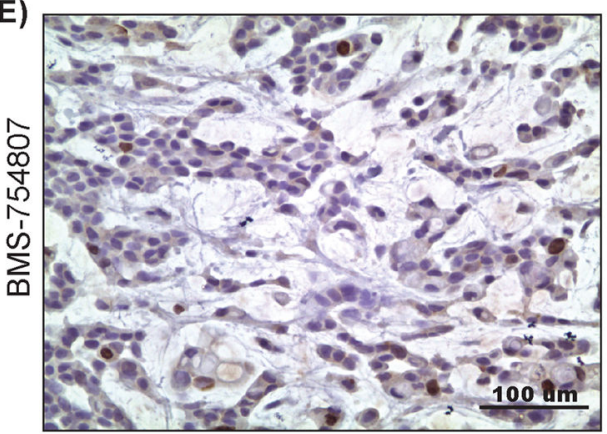

F)

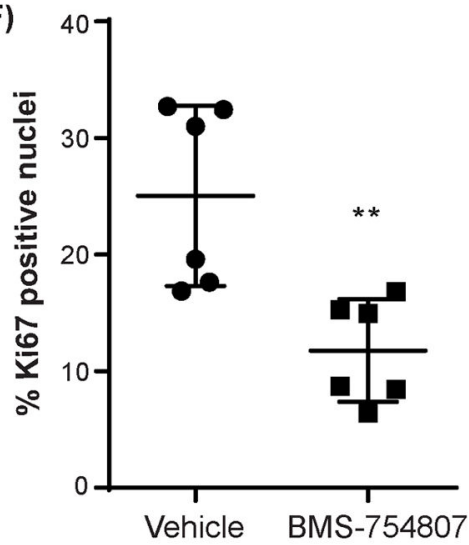

Figure 6: IGF1R/InsR inhibition reduces Ki67 staining in ILC tumor ex vivo culture.

MM134 and BCK4 xenograft tumors were harvested from immunocompromised mice, minced into $1-2 \mathrm{~mm}^{3}$ tumor chunks and then plated on gelatin sponges in 12-well plate containing $1.5 \mathrm{ml}$ media. Media was treated with DMSO Vhc or 1uM BMS-754807 for 72 hours. Tumor pieces were harvested by FFPE and stained for Ki67 as a marker of proliferation (A-B, MM134; D-E, BCK4). Staining was quantified by counting all clearly defined nuclei in 20x images ( $\mathrm{C}$ and F). Statistical difference was assessed using a Student's t-test $(\mathrm{p}<0.05 ; \mathrm{n}=3-6)$. 